\title{
Two dimensional discrete fractional Fourier transform
}

\author{
Soo-Chang $\mathrm{Pei}^{\mathrm{a}, *}$, Min-Hung Yeh ${ }^{\mathrm{b}}$ \\ ${ }^{a}$ Department of Electrical Engineering, National Taiwan University, Taipei, Taiwan, ROC \\ ${ }^{\mathrm{b}}$ Department of Computer Information Science, Tamui Oxford University College, Tamsui, Taipei, Taiwan, ROC
}

Received 4 November 1996; received in revised form 7 January 1998

\begin{abstract}
Fractional Fourier transform (FRFT) performs a rotation of signals in the time-frequency plane, and it has many theories and applications in time-varying signal analysis. Because of the importance of fractional Fourier transform, the implementation of discrete fractional Fourier transform will be an important issue. Recently, a discrete fractional Fourier transform (DFRFT) with discrete Hermite eigenvectors has been proposed, and it can provide similar results to match the continuous outputs. On the other hand, the two dimensional continuous fractional Fourier transform is also proposed for 2D signal analysis. This paper develops a 2D DFRFT which can preserve the rotation properties and provide similar results to continuous FRFT. (C) 1998 Elsevier Science B.V. All rights reserved.
\end{abstract}

\section{Zusammenfassung}

Die fraktionale Fouriertransformation (FRFT) führt eine Signaldrehung in der Zeit-Frequenz-Ebene durch, und hat viele theoretische und praktische Anwendungen bei der Analyse zeitvarianter Signale. Wegen der Wichtigkeit der fraktionalen Fouriertransformation ist die Implementierung der diskreten fraktionalen Fouriertransformation eine bedeutende Aufgabe. Kürzlich wurde eine diskrete fraktionale Fouriertransformation (DFRFT) mit diskreten Hermiteschen Eigenvektoren vorgeschlagen. Sie kann ähnliche Ergebnisse wie die zugehörigen stetigen Ausgangssignale vorweisen. Andererseits wurde auch die zweidimensionale stetige fraktionale Fouriertransformation für 2D Signalanalyse vorgeschlagen. Diese Arbeit entwickelt eine 2D DFRFT, die die Rotationseigenschaften beibehält und ähnliche Ergebnisse liefert wie die stetige FRFT. (C) 1998 Elsevier Science B.V. All rights reserved.

\section{Résumé}

La transformation de Fourier fractionnaire (FRFT) opère une rotation des signaux dans le plan temps-fréquence, et offre de nombreux concepts théoriques et applications en analyse de signaux variant dans le temps. Du fait de l'importance de la FRFT, l'implantation de la transformation de Fourier fractionnaire discrète constitue un point important. Une transformation de Fourier fractionnaire discrète (DFRFT) basée sur les vecteurs propres de Hermite discrets a récemment été proposée, et celle-ci fournit des résultats similaires á ceux du cas continu. D’autre part, la transformation de Fourier fractionnaire continue bi-dimensionnelle a également été proposée pour l'analyse des signaux 2D. Cet article développe une DFRFT 2D pouvant préserver les propriétés de rotation et fournir des résultats semblables à ceux de la FRFT continue. (C) 1998 Elsevier Science B.V. All rights reserved.

Keywords: Fourier transform; Discrete fractional Fourier transform; 2D discrete orthogonal transform

\footnotetext{
*Corresponding author. E-mail: pei@cc.ee.ntu.edu.tw.
} 


\section{Introduction}

The Fourier transform is one of the most important mathematical tools used in physical optics, linear system theory, signal processing, and so on $[2,16]$. The generalization of Fourier transform, Fractional Fourier transform (FRFT), was first introduced by Namias in $1980[11,15]$. The conventional Fourier transform can be regarded as a $\pi / 2$ rotation in the time-frequency plane, and the FRFT performs a rotation of signal to any angle. Moreover, fractional Fourier transform serves as an orthonormal signal representation for chirp signal. The fractional Fourier transform is also called rotational Fourier transform or angular Fourier transform in some documents. The properties of FRFT are well summarized in [1]. Besides being a generalization of Fourier transform, the FRFT is also related to other time-varying signal processing tools, such as Wigner distribution [6], short-time Fourier transform [6], Wavelet transform and so on [17]. The applications of FRFT are well discussed in many documents $[4,5,9,10,14]$.

The continuous FRFT is often implemented by optical instruments $[12,13]$. Because of the importance of fractional Fourier transform, its digital implementation has become an important issue in signal processing $[8,17-19,24-26]$. In the history of discrete fractional Fourier transform (DFRFT) development, the DFRFT has been considered as the linear combination of the four parts in many documents [17,24-26]. The four parts include the original signal, a circular flipped version of the signal, its DFT and a circular flipped version of its DFT. Unfortunately, this method cannot have similar outputs as the continuous FRFT. A rigorous discussion for the mismatches is presented in [18]. Besides the DFRFT in [25], an important method for digital FRFT computation has been proposed in $[8,18]$, but the angle addition property cannot be perfectly preserved. So signals can only be recovered back from their transforms within some approximation errors. In 1996, a better improvement of DFRFT was made in $[19,20]$. Pei and Yeh have found that the DFRFT with discrete Hermite eigenvectors can have similar results as those of continuous FRFT [19,20]. This DFRFT can have the mixed time and frequency character- istics of signals, which is the same as the continuous FRFT.

Several orthogonal transforms have been successfully used for two dimensional signal processing. For example, the 2D Fourier transform computes the spectrum for a 2D signal, and 2D discrete cosine transform (DCT) is widely used in image compression [7]. In order to analyze the 2D timevarying signals, the developments of $2 \mathrm{D}$ continuous and discrete fractional Fourier transforms are necessary. In [22], a continuous 2D fractional Fourier transform with various orders in the two dimensions is defined, and it is implemented by optical instruments. Until now, the development and implementation of 2D DFRFT are still not presented for 2D time-varying discrete signal analysis.

The goal of this paper is to develop 2D discrete fractional Fourier transform and discuss the properties of 2D DFRFT. This paper is organized as follows. Section 2 first reviews 1D/2D continuous FRFTs, 1D DFRFT and the theories of 2D discrete orthogonal transforms. Then the 2D DFRFT is developed. In Section 3, the 2D DFRFT for several 2D discrete signals are computed. The final conclusions of this paper are made in the last section.

\section{Development of 2D DFRFT}

In this section, the previous results used for developing 2D DFRFT are reviewed, and they include 1D FRFT, 2D FRFT, 1D DFRFT and the theories of 2D transforms. We will use these results to develop our 2D DFRFT.

\subsection{The results of $1 D$ continuous FRFT}

By successive applications of the forward Fourier transform $\mathscr{F}$ on the signal $x(t)$ several times, we will obtain

$$
\begin{aligned}
& \mathscr{F}^{2}[x(t)]=x(-t), \\
& \mathscr{F}^{3}[x(t)]=\mathscr{X}(-w), \\
& \mathscr{F}^{4}[x(t)]=x(t) .
\end{aligned}
$$

Based upon the above notation, the Fourier transform of a signal can be interpreted as a $\pi / 2$ angle 
rotation of the signal in the time-frequency plane. A generalization of Fourier transform, FRFT, is developed and treated as a rotation of the signal to arbitrary angles in the time-frequency plane, and it satisfies the following rotation properties:

1. Zero rotation: $\mathscr{R}_{0}=\boldsymbol{I}$;

2. Additivity of rotation: $\mathscr{R}_{\alpha} \mathscr{R}_{\beta}=\mathscr{R}_{\alpha+\beta}$;

3. Consistency with Fourier transform: $\mathscr{R}_{\pi / 2}=\mathscr{F}$;

4. $2 \pi$ rotation: $\mathscr{R}_{2 \pi}=\boldsymbol{I}$,

where $\mathscr{R}$ indicates the rotation operation in the time-frequency plane. $\mathscr{F}$ is the traditional Fourier transform operation. Parameters $\alpha$ and $\beta$ are the rotation angles between the transformed signal and the time axis in the time-frequency plane.

The FRFT transform kernel is defined as follows $[1,15]$ :

$$
\begin{aligned}
& K_{\alpha}(t, u)=\left\{\begin{array}{c}
\sqrt{\frac{1-j \cot \alpha}{2 \pi}} \mathrm{e}^{\mathrm{j}\left(t^{2}+u^{2}\right) / 2 \cot \alpha-\mathrm{j} u t \operatorname{cosec} \alpha} \\
\text { if } \alpha \text { is not a multiple of } \pi \\
\delta(t-u) \\
\text { if } \alpha \text { is a multiple of } 2 \pi \\
\delta(t+u) \\
\text { if } \alpha+\pi \text { is a multiple of } 2 \pi
\end{array}\right. \\
&=\sum_{n=0}^{\infty} \mathrm{e}^{-\mathrm{j} \alpha n} H_{n}(t) H_{n}(u),
\end{aligned}
$$

where $\alpha$ indicates the rotation angle of the transformed signal for FRFT. $H_{n}(t)$ is the $n$th order normalized Hermite-Gaussian function with unit variance.

$H_{n}(t)=\frac{1}{\sqrt{2^{n} n ! \sqrt{\pi}}} h_{n}(t) \mathrm{e}^{-t^{2} / 2}$,

where $h_{n}(t)$ is the $n$th order Hermite polynomial [23]. Because the Hermite-Gaussian function is an eigenfunction of the Fourier transform, Eq. (3) is treated as the eigen-decomposition of the FRFT kernel. And $\mathrm{e}^{-\mathrm{j} \alpha n}$ is the eigenvalue of continuous FRFT. Using the FRFT kernel, the FRFT of the signal $x(t)$ by angle $\alpha$ is computed as

$\mathscr{X}_{\alpha}(u)=\int_{-\infty}^{\infty} x(t) K_{\alpha}(t, u) \mathrm{d} t$.
The signal $x(t)$ can be recovered back by an FRFT operation with backward angle $(-\alpha)$ :

$x(t)=\int_{-\infty}^{\infty} \mathscr{X}_{\alpha}(u) K_{-\alpha}(u, t) \mathrm{d} u$.

\subsection{The results of $2 D$ continuous FRFT}

In [22], the 2D FRFT transform kernel with various orders in two dimensions is defined as follows:

$$
\begin{aligned}
K_{\alpha, \beta}(s, t, u, v)= & \frac{1}{2 \pi} \sqrt{1-\mathrm{j} \cot \alpha} \sqrt{1-\mathrm{j} \cot \beta} \\
& \times \exp ^{\left[\mathrm{j}\left(s^{2}+u^{2}\right) / 2 \cot \alpha-\mathrm{j} s u \operatorname{cosec} \alpha\right]} \\
& \times \exp ^{\left[\mathrm{j}\left(t^{2}+v^{2}\right) / 2 \cot \beta-\mathrm{j} t v \operatorname{cosec} \beta\right]},
\end{aligned}
$$

where $\alpha$ and $\beta$ indicate the rotation angles of the transformed signal for 2D FRFT. Using this $2 \mathrm{D}$ FRFT kernel, the 2D FRFT of the signal $x(s, t)$ by angle parameter $(\alpha, \beta)$ is computed as

$\mathscr{X}_{\alpha, \beta}(u, v)=\int_{-\infty}^{\infty} \int_{-\infty}^{\infty} x(s, t) K_{\alpha, \beta}(s, t, u, v) \mathrm{d} s \mathrm{~d} t$.

The signal $x(s, t)$ can be recovered by a 2D FRFT operation with backward angles $(-\alpha,-\beta)$ :

$$
\begin{aligned}
& x(s, t)= \\
& \int_{-\infty}^{\infty} \int_{-\infty}^{\infty} X_{\alpha, \beta}(u, v) K_{-\alpha,-\beta}(u, v, s, t) \mathrm{d} u \mathrm{~d} v .
\end{aligned}
$$

\subsection{The results of $1 D$ DFRFT}

Discrete fractional Fourier transform must obey the rotational properties as the continuous FRFT. These rotation properties can be easily realized by the power law of kernel matrix in discrete case. So the fractional power of kernel matrix is required for computing the DFRFT. In $[24,25]$, a method for computing DFRFT has been proposed by Santhanam and McClellan. And the DFRFT in $[24,25]$ is divided into four parts: the original signal, its DFT, a circular flipped version of the signal, and a circular flipped version of its DFT. Unfortunately, this DFRFT cannot have similar outputs as those 
of the continuous FRFT. A detailed discussion for the mismatches of [25] has been presented in [18].

For the development of current improved DFRFT we must thank B.W. Dickinson and K. Steiglitz [3]. They introduced a commuting matrix $\boldsymbol{S}$ to compute the real eigenvectors of the DFT kernel matrix $\boldsymbol{F}$ :

$\boldsymbol{S}=\left[\begin{array}{cccccc}2 & 1 & 0 & 0 & \cdots & 1 \\ 1 & 2 \cos \omega & 1 & 0 & \cdots & 0 \\ 0 & 1 & 2 \cos 2 \omega & 1 & \cdots & 0 \\ \vdots & \vdots & \vdots & \ddots & & \vdots \\ 1 & 0 & 0 & 0 & \cdots & 2 \cos (N-1) \omega\end{array}\right]$

where $\omega=2 \pi / N$ and $N$ is the size of the DFT kernel matrix. Matrix $\boldsymbol{S}$ commutes with matrix $\boldsymbol{F}$, and it satisfies the commutative property: $\boldsymbol{F S}=\boldsymbol{S F}$. The eigenvectors of matrix $\boldsymbol{S}$ are also the eigenvectors of matrix $\boldsymbol{F}$, but their eigenvalues are distinct. Because matrix $\boldsymbol{S}$ is a real symmetric matrix, the eigenvalues of $\boldsymbol{S}$ are all real and the eigenvectors of $\boldsymbol{S}$ are orthonormal to each other.

Although a method for computing the DFT real eigenvectors is proposed in [3], the real and orthogonal eigenvectors were not used for further research and practical applications. In [19,20], Pei and Yeh used the DFT eigenvectors obtained from matrix $\boldsymbol{S}$ to construct the DFRFT kernel. The eigenvectors of matrix $\boldsymbol{S}$ are treated as discrete Hermite functions in [19]. In addition to the DFT Hermite eigenvectors, the eigenvalues and an eigenvalues assignment rule are also required for the DFRFT kernel construction. The eigenvalues assignment rule is developed similar to the notation in Eq. (3), and it is shown again in Table 1 . Such an assignment rule

\section{Table 1}

The eigenvalues assignment rule for DFRFT kernel matrix

\begin{tabular}{ll}
\hline$N$ & The eigenvalues \\
\hline $4 m$ & $\mathrm{e}^{-\mathrm{j} k \alpha}, k=0,1,2, \ldots,(4 m-2), 4 m$ \\
$4 m+1$ & $\mathrm{e}^{-\mathrm{j} k \alpha}, k=0,1,2, \ldots,(4 m-1), 4 m$ \\
$4 m+2$ & $\mathrm{e}^{-\mathrm{j} k \alpha}, k=0,1,2, \ldots, 4 m,(4 m+2)$ \\
$4 m+3$ & $\mathrm{e}^{-\mathrm{j} k \alpha}, k=0,1,2, \ldots,(4 m+1),(4 m+2)$
\end{tabular}

can make the constructed kernel consistent with an identity transform when $\alpha=0$ and a DFT while $\alpha=\pi / 2$.

After the eigenvalues and eigenvectors of the DFT kernel matrix are determined, the transformation kernel of DFRFT can be easily defined by determining the fractional powers of the eigenvalues. The transform kernel of DFRFT is computed as

$$
\begin{aligned}
\mathscr{R}_{\alpha}= & \boldsymbol{F}^{2 \alpha / \pi}=\boldsymbol{V} \boldsymbol{D}^{2 \alpha / \pi} \boldsymbol{V}^{\mathrm{T}} \\
= & \left\{\begin{array}{c}
\sum_{k=0}^{N-1} \mathrm{e}^{-\mathrm{j} k \alpha} \boldsymbol{v}_{k} \boldsymbol{v}_{k}^{\mathrm{T}} \\
\text { for } N=4 m+1,4 m+3, \\
\sum_{k=0}^{N-2} \mathrm{e}^{-\mathrm{j} k \alpha} \boldsymbol{v}_{k} \boldsymbol{v}_{k}^{\mathrm{T}}+\mathrm{e}^{-\mathrm{j} N \alpha} \boldsymbol{v}_{N-1} \boldsymbol{v}_{N-1}^{\mathrm{T}} \\
\text { for } N=4 m, 4 m+2,
\end{array}\right.
\end{aligned}
$$

where $\boldsymbol{v}_{k}$ is the $k$ th order DFT Hermite eigenvector. $\boldsymbol{V}=\left[\boldsymbol{v}_{0}\left|\boldsymbol{v}_{1}\right| \ldots \mid \boldsymbol{v}_{N-1}\right]$. Matrix $\boldsymbol{D}$ is a diagonal matrix, in which the diagonal entries have the same eigenvalues corresponding to the column eigenvectors of matrix $\boldsymbol{V}$ in its diagonal entries.

After the transformation kernel being determined, the DFRFT of signal $\boldsymbol{x}$ can be computed through Eq. (13):

$\boldsymbol{X}_{\alpha}=\mathscr{R}_{\alpha} \boldsymbol{x}=\boldsymbol{F}^{2 \alpha / \pi} \boldsymbol{x}=\boldsymbol{V} \boldsymbol{D}^{2 \alpha / \pi} \boldsymbol{V}^{\mathrm{T}} \boldsymbol{x}$.

Similar to the continuous FRFT, the signal $\boldsymbol{x}$ can also be recovered back from its DFRFT by a reverse operation with parameter $(-\alpha)$ :

$\boldsymbol{x}=\mathscr{R}_{-\alpha} \boldsymbol{X}_{\alpha}=\boldsymbol{V} \boldsymbol{D}^{-2 \alpha / \pi} \boldsymbol{V}^{\mathrm{T}} \boldsymbol{X}_{\alpha}$

Fig. 1 shows the outputs of a rectangular window for the three methods: continuous FRFT, DFRFT in [25] and DFRFT in [19]. The mismatches in the results of [25] are very clear. Our DFRFT has made great improvements and good matches with the continuous cases.

The method in [19] can have similar results as those of continuous FRFT, but the eigenvectors of matrix $\boldsymbol{S}$ are not the optimal solution for DFT 

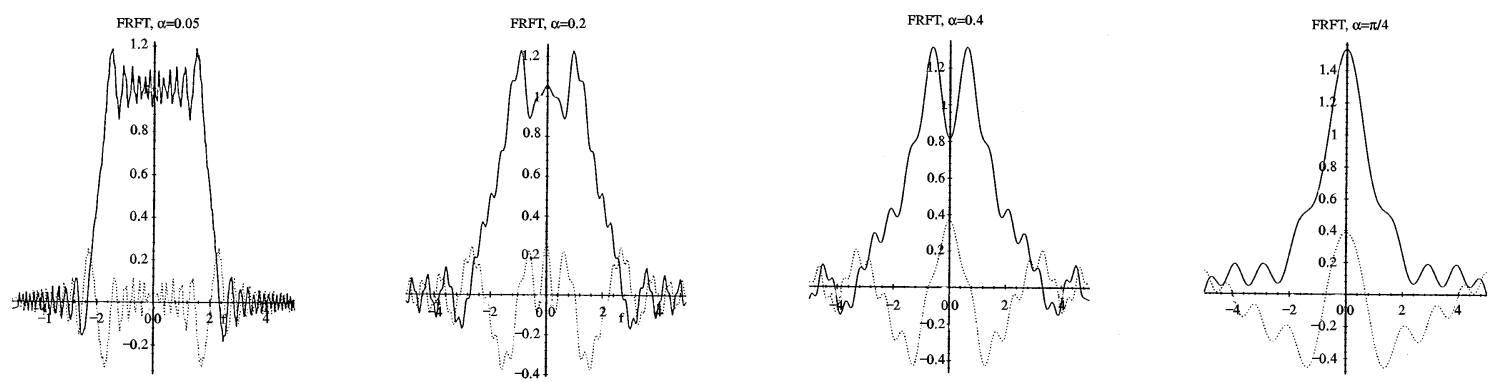

(a) Transform outputs of continuous FRFT.
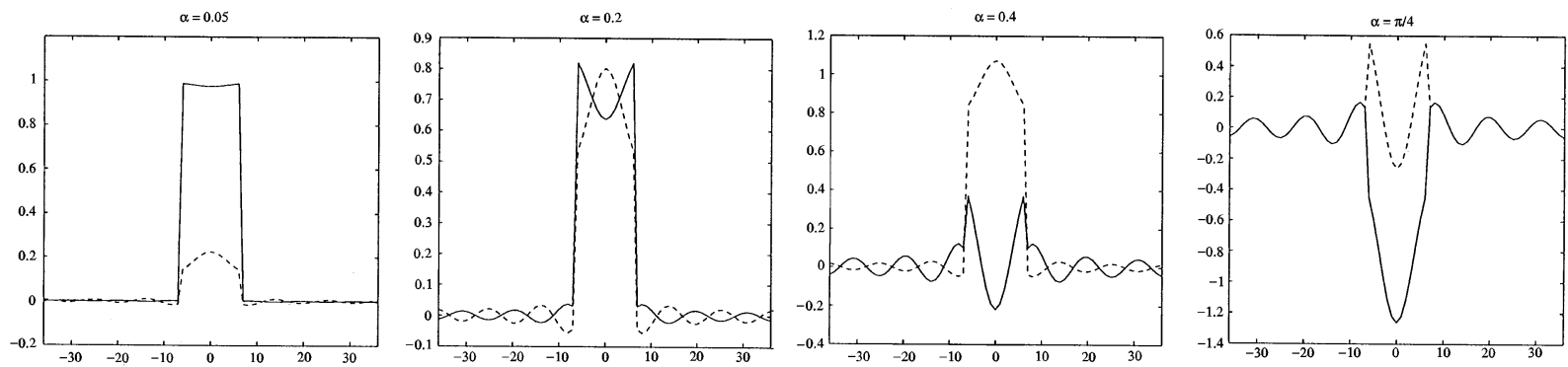

(b) Transform outputs of DFRFT in [25].
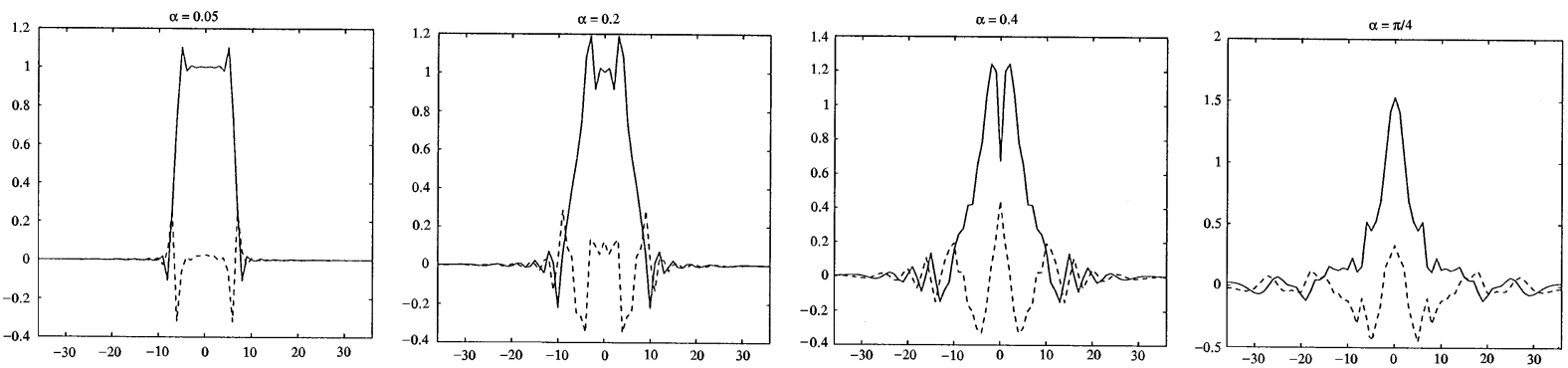

(c) Transform outputs of DFRFT in [19].

Fig. 1. The comparison of three transform methods for a rectangular window.

Hermite eigenvectors. There still exists spaces for further researches and improvements.

\subsection{Development of the $2 D$ DFRFT}

Several two dimensional unitary transforms have been used in signal processing, such as discrete cosine transform [7], discrete Walsh transform [7], and so on. The $(M, N)$-point $2 \mathrm{D}$ unitary discrete transform is computed as
$\boldsymbol{X}(m, n)=\sum_{p=0}^{M-1} \sum_{q=0}^{N-1} \boldsymbol{x}(p, q) \boldsymbol{K}(p, q, m, n)$,

where $\boldsymbol{K}(p, q, m, n)$ is the $2 \mathrm{D}$ transform kernel. If $\boldsymbol{K}=\boldsymbol{K}_{1} \otimes \boldsymbol{K}_{2}$, then the transform kernel $\boldsymbol{K}(p, q, m, n)$ is called separable [7], where $\otimes$ denotes the tensor product. For a 2D separable kernel, its 2D transform can be implemented by row-column computation:

$\boldsymbol{X}(m, n)=\sum_{p=0}^{M-1}\left[\sum_{q=0}^{N-1} \boldsymbol{x}(p, q) \boldsymbol{K}_{2}(q, n)\right] \boldsymbol{K}_{1}(p, m)$. 
Table 2

Properties of 2D DFRFT

\begin{tabular}{ll}
\hline Unitary & $\mathscr{R}_{(\alpha, \beta)}^{*}=\mathscr{R}_{(\alpha, \beta)}^{-1}=\mathscr{R}_{(-\alpha,-\beta)}$ \\
\hline Angle additivity & $\mathscr{R}_{\left(\alpha_{1}, \beta_{1}\right)} \mathscr{R}_{\left(\alpha_{2}, \beta_{2}\right)}=\mathscr{R}_{\left(\alpha_{1}+\beta_{1}, \alpha_{2}+\beta_{2}\right)}$ \\
Time reversal & $\mathscr{R}_{(\alpha, \beta)} \boldsymbol{x}(-m,-n)=\boldsymbol{X}_{(\alpha, \beta)}(-m,-n)$ \\
DFT rotation & $\mathscr{R}_{\left(\alpha+\frac{\pi}{2}, \beta+\frac{\pi}{2}\right)}=\boldsymbol{F} \mathscr{R}_{(\alpha, \beta)}$ \\
Periodicity & $\mathscr{R}_{(\alpha+2 \pi, \beta+2 \pi)}=\mathscr{R}_{(\alpha+2 \pi, \beta)}=\mathscr{R}_{(\alpha, \beta+2 \pi)}=\mathscr{R}_{(\alpha, \beta)}$ \\
Parity & If $\boldsymbol{x}(m, n)$ is even, $\boldsymbol{X}_{(\alpha, \beta)}(m, n)$ is even \\
& If $\boldsymbol{x}(m, n)$ is odd, $\boldsymbol{X}_{(\alpha, \beta)}(m, n)$ is odd \\
Eigenfunction & $\mathscr{R}_{(\alpha, \beta)}\left[\boldsymbol{v}_{s, t}\right]=\mathrm{e}^{-\mathrm{j} \alpha s} \mathrm{e}^{-\mathrm{j} \beta \boldsymbol{t}} \boldsymbol{v}_{s, t}$ \\
& $\boldsymbol{v}_{s, t}$ is the $(s, t)$-order Hermite-like eigenfunc- \\
& tion
\end{tabular}

can also preserve the desired properties of continuous 2D FRFT: Table 2 summarizes the properties of 2D DFRFT.

Because the transform kernel of 2D DFRFT is defined as a separable form, the 2D DFRFT can be implemented by row-column computation. This method can be applied to any 2D discrete fractional transforms, such as 2D discrete fractional Hartley transform [21] and other discrete unitary fractional transforms which may probably be developed in the future.

\section{Experimental results}

In [22], the 2D continuous FRFT transform kernel is separable. So the 2D DFRFT is also defined with a separable form. Thus it is defined as

$\mathscr{R}_{(\alpha, \beta)}=\mathscr{R}_{\alpha} \otimes \mathscr{R}_{\beta}$,

where $\mathscr{R}_{\alpha}, \mathscr{R}_{\beta}$ are the 1D DFRFT transform kernel proposed in $[19,20]$. These two parameters in DFRFT, $\alpha$ and $\beta$, indicate the individual fractional orders in two dimensions. Then the forward and inverse 2D DFRFT are computed as

$\boldsymbol{X}_{(\alpha, \beta)}(m, n)=\sum_{p=0}^{M-1} \sum_{q=0}^{N-1} \boldsymbol{x}(p, q) \mathscr{R}_{(\alpha, \beta)}(p, q, m, n)$,

$\boldsymbol{x}(p, q)=\sum_{m=0}^{M-1} \sum_{n=0}^{N-1} \boldsymbol{X}_{(\alpha, \beta)}(m, n) \mathscr{R}_{(-\alpha,-\beta)}(p, q, m, n)$.

The special 2D DFRFTs are discussed in the following:

1. If $\alpha=\beta=\pi / 2$, the 2D DFRFT performs the conventional 2D DFT.

2. If $\alpha=\pi / 2$ and $\beta=0$, the 2D DFRFT performs only row DFT transforms.

3. If $\alpha=0$ and $\beta=\pi / 2$, the 2D DFRFT performs only column DFT transforms.

4. If $\alpha=\beta=0$, the 2D DFRFT performs the identity transform (no transform).

The 1D DFRFT proposed in $[19,20]$ can preserve the desired properties of FRFT, so this 2D DFRFT
In this section, we will apply the proposed 2D DFRFT algorithm to compute the transforms of

Example 1. In this example, we will compute the 2D DFRFT for a $2 \mathrm{D}$ discrete rectangular window. Fig. 2 shows the amplitudes of 2D DFRFT of a rectangular window for various angular parameters. The $2 \mathrm{D}$ rectangular window used in this example is a $37 \times 37$ discrete signal. $f(x, y)=1$, for $-4 \leqslant x \leqslant 4$ and $-4 \leqslant y \leqslant 4$; otherwise, $f(x, y)=0$. From the results shown in Fig. 2, it is obvious to find out that the transform results are changing from the $2 \mathrm{D}$ rectangular window to a $2 \mathrm{D}$ sinc function while both the angular parameters change from 0 to $\pi / 2$. The upper-right and lower-left plots of Fig. 2 are the 2D DFRFT which are performed only the row transforms and the column transforms, respectively.

Example 2. The 2D DFRFT for a 2D discrete circular window with various orders are computed in this example. Fig. 3 shows the amplitudes of 2D DFRFT of a circular window for various angular parameters. The $2 \mathrm{D}$ circular window used in this example is a $37 \times 37$ discrete signal. $f(x, y)=1$, for $x^{2}+y^{2} \leqslant 25$; otherwise $f(x, y)=0$. Fig. 3 clearly shows that the transform results are varying from the $2 \mathrm{D}$ circular window to its $2 \mathrm{D}$ DFT output. The upper-right and lower-left plots of Fig. 2 are the 2D DFRFT which are performed only the row transforms and the column transforms. several practical 2D discrete signals. 
$\alpha=0, \beta=0$

$\alpha=0, \beta=\pi / 6$

$\alpha=0, \beta=\pi / 3$

$\alpha=0, \beta=\pi / 2$

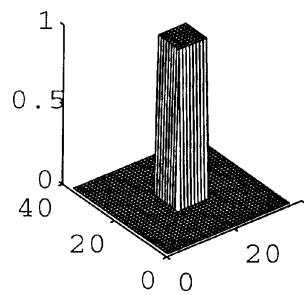

$\alpha=\pi / 6, \beta=0$

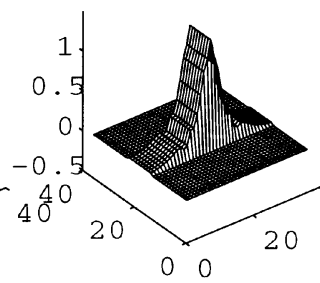

$\alpha=\pi / 6, \beta=\pi / 6$

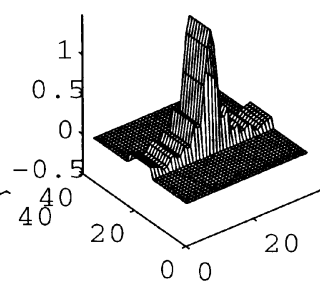

$\alpha=\pi / 6, \beta=\pi / 3$

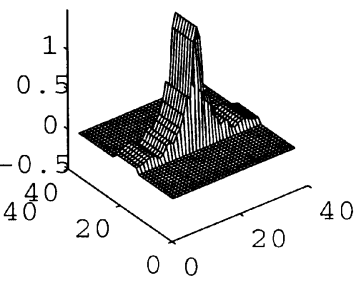

$\alpha=\pi / 6, \beta=\pi / 2$
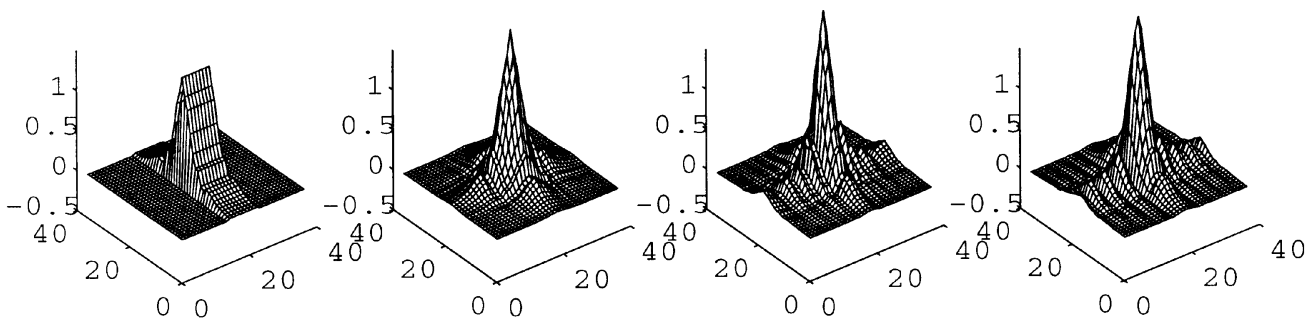

$\alpha=\pi / 3, \beta=0$

$\alpha=\pi / 3, \beta=\pi / 6$

$\alpha=\pi / 3, \beta=\pi / 3$

$\alpha=\pi / 3, \beta=\pi / 2$
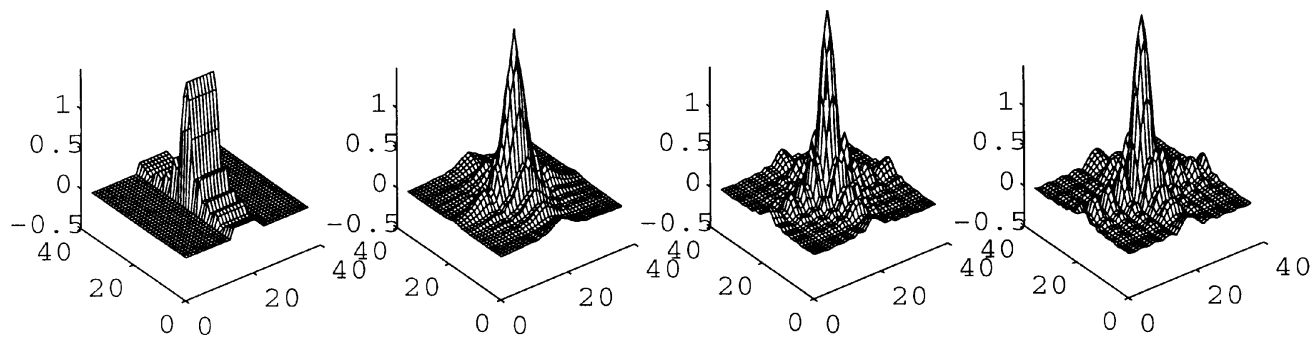

$\alpha=\pi / 2, \beta=0$

$\alpha=\pi / 2, \beta=\pi / 6$

$\alpha=\pi / 2, \beta=\pi / 3$

$\alpha=\pi / 2, \beta=\pi / 2$
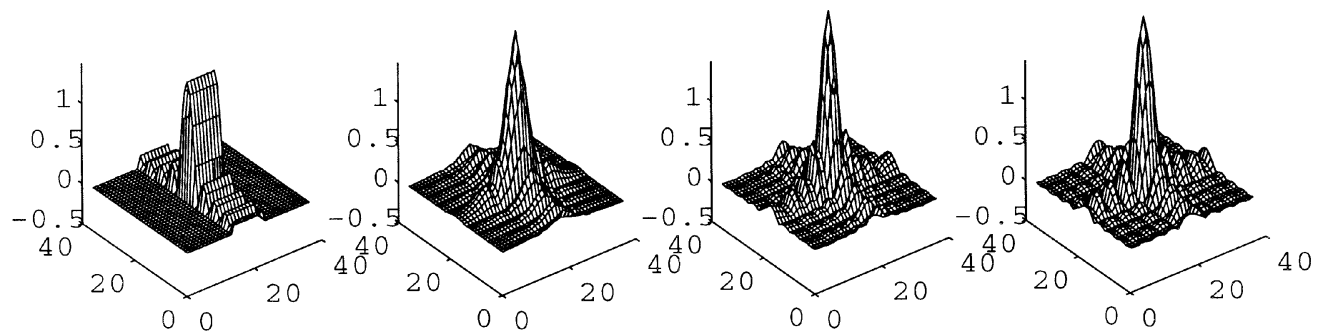

Fig. 2. 2D DFRFT of the rectangular window in Example 1.

Example 3. In this example, we compute the 2D DFRFT for a digital image. Fig. 4 shows the amplitudes of 2D DFRFT of this image. The upper-left plot of Fig. 4 is the original image, and the lowerright plot is the conventional 2D DFT of this image. The parameters, $\alpha$ and $\beta$, used in this example are identical. It can be found that the transform results are varying from the original signal to its $2 \mathrm{D}$ DFT while the angles vary from 0 to $\pi / 2$.

\section{Conclusions}

The present work has developed a 2D DFRFT which can be realized in digital computers. This 2D 

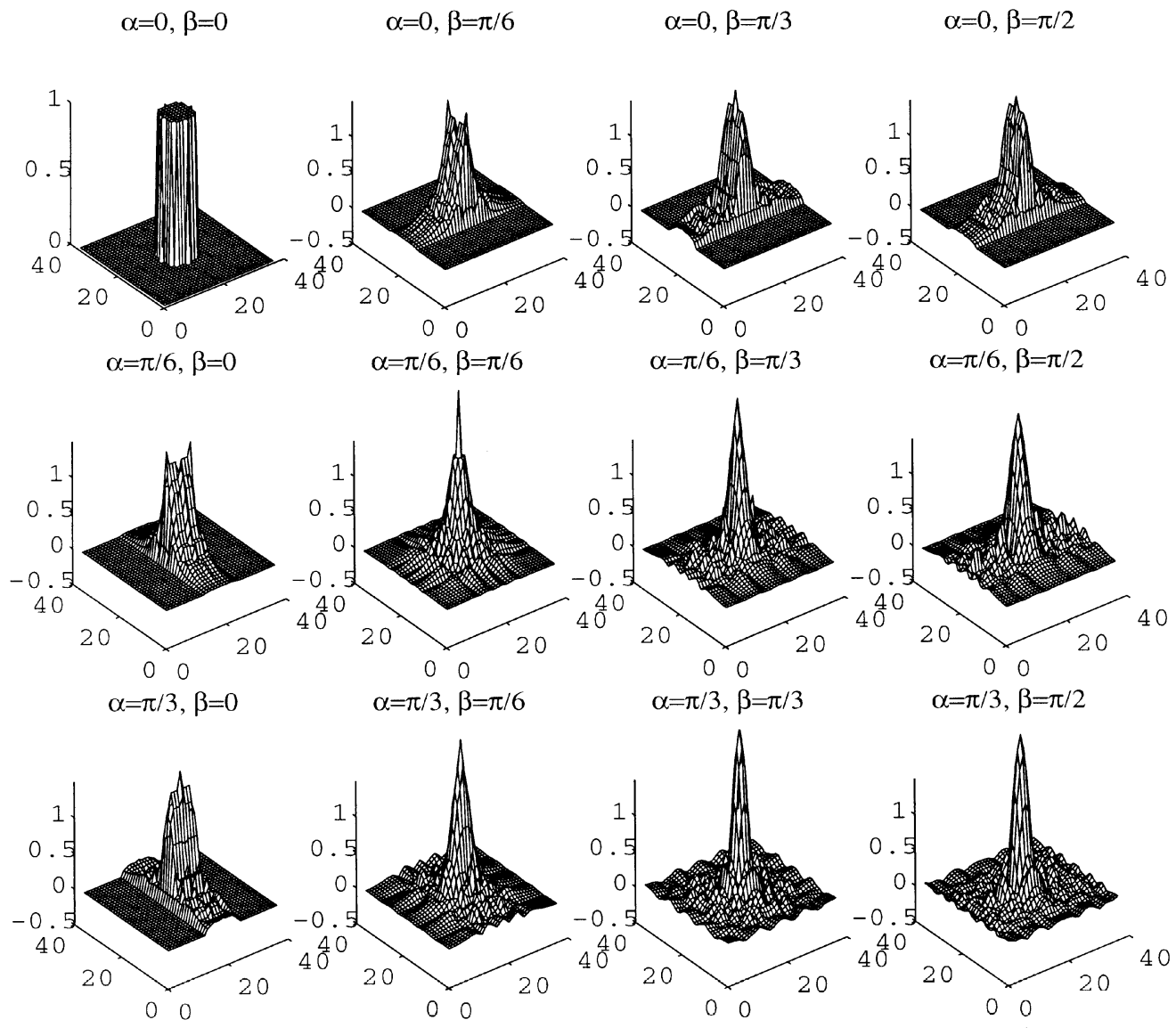

$$
\alpha=\pi / 2, \beta=0
$$
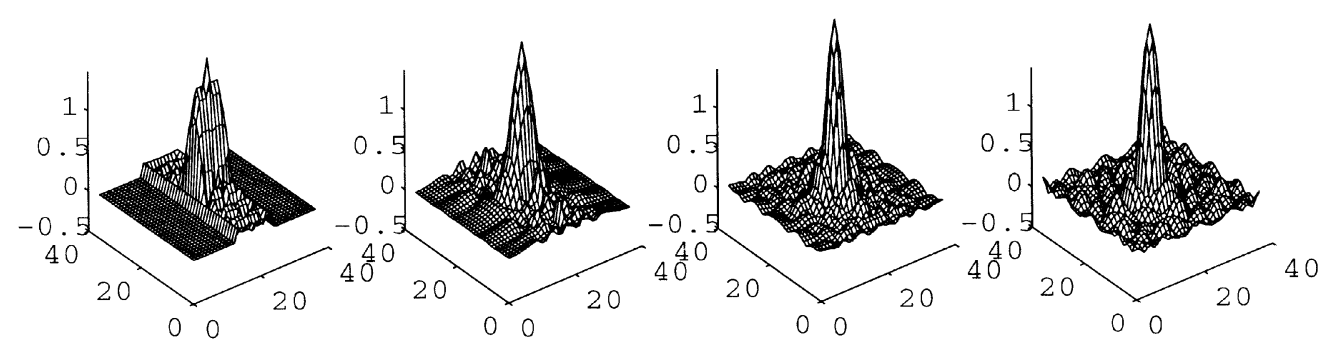

Fig. 3. 2D DFRFT of the circular window in Example 2.

DFRFT can preserve the rotation properties and provide similar results to the corresponding continuous case. The mixed time and frequency characteristics of signals are revealed in the transform outputs. So this 2D DFRFT can be used for 2D digital time-varying signal analysis. More- over, the implementation method of 2D DFRFT can be realized by the row-column computation which is the same as other unitary transforms. The separable scheme in the 2D DFRFT can be applied to other 2D unitary fractional transforms. 
$\alpha=\beta=0$

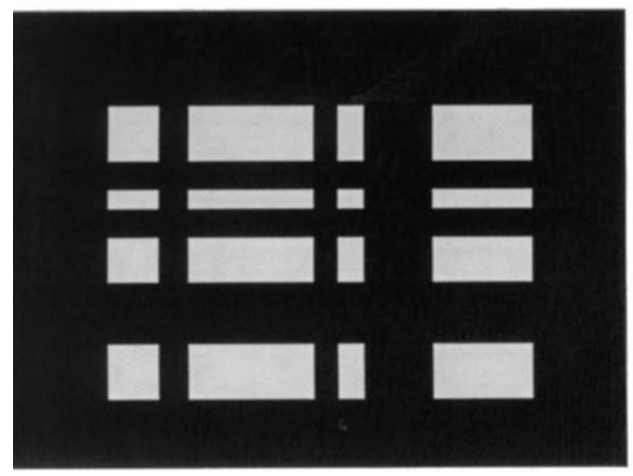

$\alpha=\beta=\pi / 5$

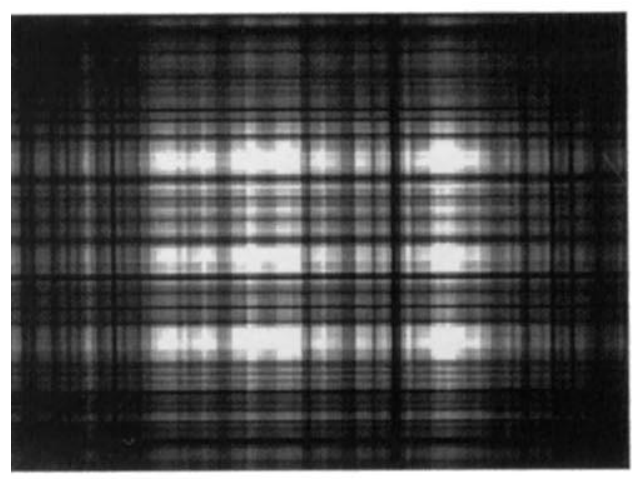

$\alpha=\beta=2 \pi / 5$

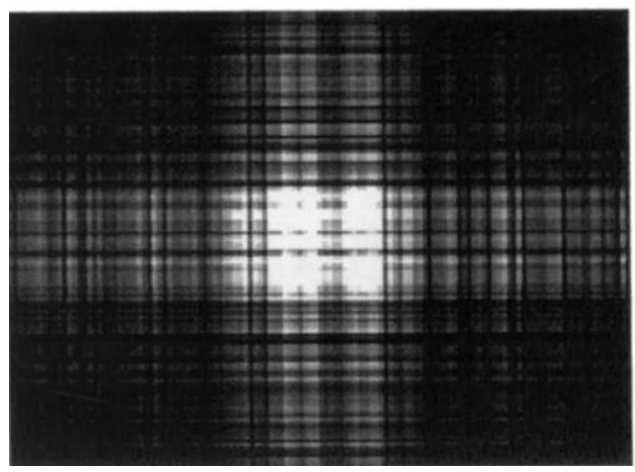

$\alpha=\beta=\pi / 10$

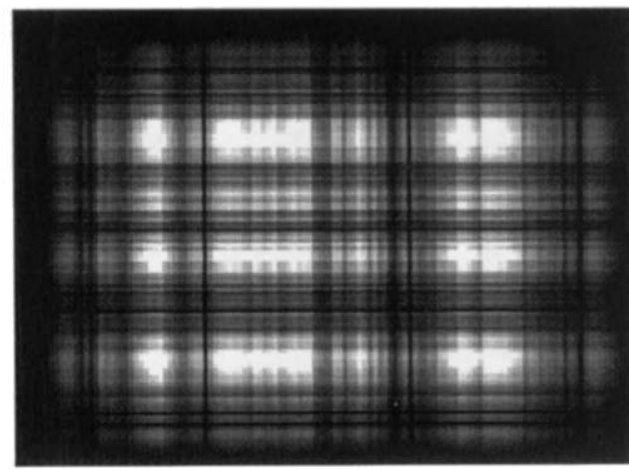

$\alpha=\beta=3 \pi / 10$

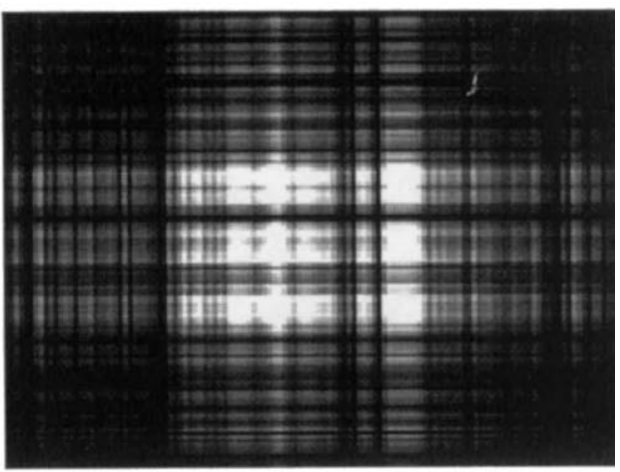

$\alpha=\beta=\pi / 2$

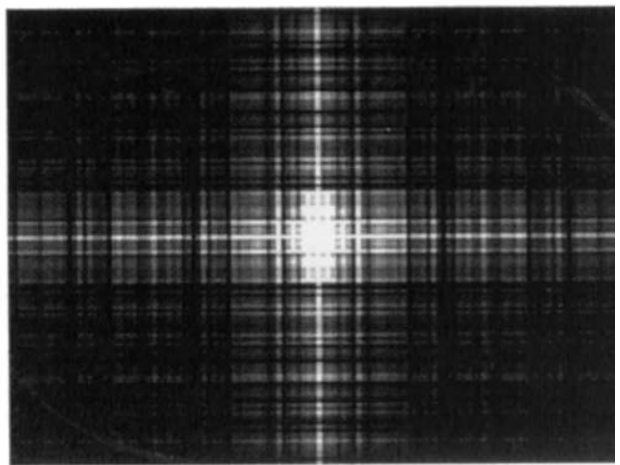

Fig. 4. 2D DFRFT of the image in Example 3. 


\section{References}

[1] L.B. Almeida, The fractional Fourier transform and time-frequency representation, IEEE Trans. Signal Process. 42 (November 1994) 3084-3091.

[2] R.N. Bracewell, The Fourier Transform and its Applications, 2nd ed., McGraw-Hill, New York, 1986.

[3] B.W. Dickinson, K. Steiglitz, Eigenvectors and functions of the discrete Fourier transform, IEEE Trans. Acoust. Speech Signal Process. ASSP-30 (February 1982) 25-31.

[4] R.G. Dorsch, A.W. Lohmann, Y. Bitran, D. Mendlovic, Chirp filtering in the fractional Fourier domain, Appl. Opt. 33 (1994) 7599-7602.

[5] D. Dragonman, Fractional Wigner distribution function, J. Opt. Soc. Amer. A 13 (March 1996) 474-478.

[6] F. Hlawatsch, G.F. Bourdeaux-Bartels, Linear and quadratic time-frequency signal representations, IEEE Signal Process. Mag. 9 (April 1992) 21-67.

[7] A.K. Jain, Fundamentals of Digital Image Processing. Prentice-Hall, Englewood Cliffs, NJ, 1989.

[8] M.A. Kutay, H.M. Ozaktas, L. Onural, O. Arikan, Optimal Filtering in fractional Fourier domains, in: Proc. IEEE Internat. Conf. on Acoustics, Speech, and Signal Processing, 1995, pp. 937-940.

[9] A.W. Lohmann, Image rotation, Wigner rotation, and the fractional Fourier transform, J. Opt. Soc. Amer. A 10 (1993) 2181-2186.

[10] A.W. Lohmann, B.H. Soffer, Relationships between the Radon-Wigner and fractional Fourier transforms, J. Opt. Soc. Amer. A 11 (June 1994) 1798-1801.

[11] A.C. McBride, F.H. Kerr, On Namias' fractional Fourier transforms, IMA J. Appl. Math. 39 (1987) 159-175.

[12] D. Mendlovic, H.M. Ozaktas, Fractional Fourier transformations and their optical implementation: I, J. Opt. Soc. Amer. A 10 (1993) 1875-1881.

[13] D. Mendlovic, H.M. Ozaktas, Fractional Fourier transformations and their optical implementation: II, J. Opt. Soc. Amer. A 10 (1993) 2522-2531.
[14] D. Mendlovic, H.M. Ozaktas, A.W. Lohmann, Fractional correlation, Appl. Opt. 34 (January 1995) 303-309.

[15] V. Namias, The fractional order Fourier transform and its application to quantum mechanics, J. Inst. Math. Appl. 25 (1980) 241-265.

[16] A.V. Oppenheim, Discrete-time Signal Processing, Prentice-Hall, Englewood Cliffs, NJ, 1989.

[17] H.M. Ozaktas, B. Barshan, D. Mendlovic, L. Onural, Convolution, filtering, and multiplexing in fractional Fourier domains and their relationship to chirp and wavelet transforms, J. Opt. Soc. Amer. A 11 (February 1994) 547-559.

[18] H.M. Ozaktas, O. Arikan, M.A. Kutay, G. Bozdagi, Digital computation of the fractional Fourier transform, IEEE Trans. on Signal Process. 44 (September 1996) 2141-2150.

[19] S.C. Pei, M.H. Yeh, Discrete fractional Fourier transform, in: Proc. IEEE Internat. Symp. on Circuits and Systems, May 1996, pp. 536-539.

[20] S.C. Pei, M.H. Yeh, Improved discrete fractional fourier transform, Opt. Lett. 22 (July 1997) 1047-1049.

[21] S.C. Pei, C.C. Tseng, M.H. Yeh, J.J. Shyu, Discrete fractional Hartley and Fourier transforms, IEEE Trans. Circuit and System, Part II, to appear.

[22] A. Sahlin, H.M. Ozaktas, D. Mendlovic, Optical implementation of the two-dimensional fractional Fourier transform with different orders in the two dimensions, Opt. Commun. 120 (1995) 134-138.

[23] G. Sansone, Orthogonal Functions, Interscience, New York, 1959.

[24] B. Santhanam, J.H. McClellan, The DRFT - A rotation in time-frequency space, in: Proc. IEEE Internat. Conf. on Acoustics, Speech, and Signal Processing, 1995, pp. 921-924.

[25] B. Santhanam, J.H. McClellan, The discrete rotational Fourier transform, IEEE Trans. Signal Process. 42 (April 1996) 994-998.

[26] C.C. Shih, Fractionalization of Fourier transform, Opt. Commun. 118 (August 1995) 495-498. 